\title{
ALTERAÇÕES NAS CONCENTRAÇÕES SÉRICAS DE GLICOSE, SÓDIO, POTÁSSIO, URÉIA E CREATININA, EM EQÜINOS SUBMETIDOS A PROVAS DE ENDURO DE 3OKM COM VELOCIDADE CONTROLADA
}

\author{
ALTERATIONS OF SERIC GLUCOSE, SODIUM, POTASSIUM, UREA AND CREATININE \\ LEVELS IN EQUINES SUBMITTED TO ENDURANCE RACE OF 30KM \\ WITH CONTROLED SPEED
}

\author{
Wilson Roberto Fernandes ${ }^{1}$ Maria Helena Matiko Akao Larsson ${ }^{2}$
}

RESUMO

\begin{abstract}
Foram estudadas as alterações dos parâmetros de alguns constituintes da bioquímica sérica em eqüinos submetidos a provas de enduro de $30 \mathrm{~km}$ de percurso, com velocidade controlada de 12 a $14 \mathrm{~km} / \mathrm{h}$, realizadas no Estado de São Paulo. Para tanto, foram utilizados 45 eqüinos adultos (de 4 a 10 anos de idade), divididos em três grupos, de acordo com as características raciais, da seguinte forma: grupo I constituído por 15 eqüinos da raça Árabe, grupo II, por 15 eqüinos da raça Mangalarga e grupo III, por 15 eqüinos mestiços, sendo que, nos três grupos, 11 dos animais eram machos e 4 fêmeas. As amostras de sangue foram obtidas em três tempos, a saber: antes do início da prova, 20 a 30 minutos após o seu término e 80 a 90 minutos após a realização da prova, com os animais já em repouso. As variações observadas nos valores séricos de potássio, glicose e creatinina foram semelhantes nos três grupos e apresentaram diferenças estatisticamente significativas quanto ao tempo; com relação à variação do sódio sérico, o grupo composto por animais da raça Árabe não se comportou de maneira semelhante aos demais, porém, para os três grupos, foi observada significância estatística.Todos os grupos apresentaram o mesmo padrão de variação para uréia, porém, só se comprovou diferença significante nos teores de uréia para os animais da raça Mangalarga e para os Mestiços.
\end{abstract}

Palavras-chave: eqüinos, enduro, bioquímica sérica

\section{SUMMARY}

Some plasmatic biochemical parameters of equines submitted to an endurance race of $30 \mathrm{~km}$, with controled speed between 12 to $14 \mathrm{~km} / \mathrm{h}$, taken place at State of São Paulo, Brazil, were studied. Forty five adult equines (4 to 10 years old) were used. They were divided into three groups, according to the breed features: group I: 15 Arabian animals; group II: 15 Mangalarga animals; group III: 15 mixed breed animals. Each group was constituted by 11 males and 4 females. Blood samples were collected in three different times: before the race, 20 to 30 minutes and 80 to 90 minutes after the race, with the animal resting. Seric values of potassium, glucose and creatinine were similar in all groups but there was statistical significant differences according to time collection. Concerning to sodium, all groups showed a variation statistically significcant but the Arabian equines presented a different variation pattern from the other two groups. All groups showed the same variation pattern for seric urea but significant differences for seric urea levels were observed between Mangalarga and mixed breed animals.

Key words: equine, endurance, biochemistry.

\section{INTRODUÇÃO}

O enduro eqüestre, na sua forma atual, surgiu nos Estados Unidos há cerca de quatro décadas, e somente no final da década de 80 , foi introduzido no Brasil. Eqüinos da raça Árabe são os mais utilizados, provavelmente devido à sua distribuição cosmopolita. No Brasil, além desses, são utilizados também os da raça Mangalarga e os sem raça definida que, sem dúvida, são os animais mais comuns em nosso meio.

\footnotetext{
${ }^{1}$ Médico Veterinário, Professor, Doutor do Departamento de Clínica Médica da Faculdade de Medicina Veterinária e Zootecnia (FMVZ), Universidade de São paulo (USP).

2 Médica Veterinária, Professora Associada, Departamento de Clínica Médica, FMVZ, USP, Av. Prof. Orlando Marques Paiva 87, Cidade Universitária, Butantã,São Paulo,SP 05508-000.
} 
WILLIANSON (1974), trabalhando com cavalos de corrida, observou que os animais vencedores apresentavam valores médios de sódio e potássio de 141 e $2,8 \mathrm{mEq} / \ell$, respectivamente, e naqueles que apresentavam valores abaixo de $125 \mathrm{mEq} / \ell$ de sódio e $3,6 \mathrm{mEq} / \ell$ de potássio (considerados como os limites inferiores de normalidade) podia-se notar a queda de rendimento e até mesmo alterações no traçado eletrocardiográfico relacionados à concentração sérica de potássio. Em 1978, LUCKE \& HALL, trabalhando com eqüinos de enduro em provas de $80 \mathrm{~km}$, constataram diminuição da concentração sérica de potássio, da ordem de $10 \%$, e de glicose (40\%), prova essa realizada em temperatura baixa (entre 3,5 e $8^{\circ} \mathrm{C}$ ) e velocidade média de $13,4 \mathrm{~km} / \mathrm{h}$.

ROSE et al. (1980b) avaliaram as perdas de sódio e potássio em eqüinos que cumpriram provas de $100 \mathrm{~km}$, em temperatura ambiente variando de 16 a $30^{\circ} \mathrm{C}$, mensurando esses elementos no soro e no suor. Nessa pesquisa, os autores concluíram que as perdas de potássio influenciavam significativamente o desempenho dos animais, visto que há diferença estatisticamente significante na concentração do referido eletrólito nos eqüinos que terminaram a prova $(4,00 \pm 0,11 \mathrm{mmol} / \ell)$, quando comparados aos que não conseguiam cumprir o percurso (3,60 $\pm 0,07 \mathrm{mmol} / \ell)$. ROSE et al. (1980a) fizeram avaliações seriadas de sódio, potássio, uréia, creatinina e glicose em dezesseis eqüinos Puro Sangue Inglês, que competiam na prova de "três dias". Nesse trabalho, os autores observaram que todos os elementos avaliados apresentavam aumento das suas concentrações plasmáticas durante e/ou após o término da prova, porém esse aumento não ocorria ao mesmo tempo para todos os elementos.

SNOW et al. (1982) trabalharam com quatro eqüinos que competiram em prova de Enduro de $80 \mathrm{~km}$, com velocidade de 16 a $18 \mathrm{~km} / \mathrm{h}$, determinando glicose, uréia, creatinina, sódio e potássio séricos. Observaram que, no início da prova, havia aumento da glicose sérica com posterior diminuição, a partir da metade do percurso. A uréia e a creatinina aumentavam, gradativamente, durante o exercício e iniciavam período de declínio logo após o final da prova, no caso da creatinina e depois de quatro horas de descanso, no caso da uréia. As concentrações séricas de sódio só aumentaram de forma estatisticamente significante nos últimos $16 \mathrm{~km}$ de prova até a primeira meia hora de repouso; e o potássio diminuiu significativamente no período de descanso, quando comparado com os valores obtidos durante todo o percurso. ROSE $\boldsymbol{e t}$ al. (1983) voltaram a estudar o efeito do treinamento em alguns constituintes séricos, porém, desta feita, padronizando, para os quatro eqüinos do experimento, o mesmo tipo de exercício durante sete semanas, e assim puderam afirmar que o exercício determina mudança nos valores absolutos dos elementos estudados; porém, o tipo de alteração relativa permaneceu inalterado durante as sete semanas de treinamento, para glicose, potássio e creatinina. A uréia foi o único elemento que apresentou aumento absoluto, significativo, da ordem de $12 \%$, a partir da quinta semana de treinamento.

OOSTERBAAN et al. (1991) publicaram os dados obtidos em cinco anos de uma mesma prova de enduro, em que foram analisados um total de 91 eqüinos, considerando-se tanto vencedores das provas como desclassificados. Nesse trabalho, os autores observaram aumento das concentrações de sódio e potássio durante a prova, com posterior diminuição no período de repouso. Com relação à glicose, os autores relataram aumento quando da colheita após $30 \mathrm{~km}$ de percurso e diminuição nos 60 e $80 \mathrm{~km}$, com normalização ao final da prova.

Devido à escassez de conhecimentos relativos ao desempenho de eqüinos submetidos às provas de longa duração, principalmente pelo fato dessas provas terem sido recém instituídas no país, realizou-se o presente trabalho com o objetivo de averiguar as alterações que ocorrem em alguns parâmetros da bioquímica sérica de eqüinos participantes de enduro.

\section{MATERIAL E MÉTODOS}

Utilizaram-se 45 eqüinos, machos e fêmeas, com idades variando entre 4 e 10 anos, participantes de provas de enduro, realizadas nos municípios de Itu, Pirassununga, Serra Negra, Sorocaba e Tremembé, no Estado de São Paulo, em trilhas previamente demarcadas com $30 \mathrm{~km}$ de extensão e percorridas à velocidade de 10 a $12 \mathrm{~km} / \mathrm{h}$ ora. Os eqüinos utilizados no presente trabalho foram divididos em três grupos de 15 animais, de acordo com a caracterização racial: grupo I (animais da raça Árabe), grupo II (animais da raça Mangalarga) e grupo III (animais SRD).

Somente foram considerados os resultados obtidos de animais classificados. De cada um dos eqüinos, foram colhidas amostras de sangue, por venóclise na jugular, em tubos "Vacutainer" sem anticoagulante, utilizando agulhas $40 \times 9 \mathrm{~mm}$, em três tempos distintos sendo: o tempo zero $\left(\mathrm{T}_{1}\right)$ poucos minutos antes do início da prova; tempo dois $\left(\mathrm{T}_{2}\right)$ - de 20 a 30 minutos após o término da prova e o tempo três $\left(\mathrm{T}_{3}\right)$ - de 80 a 90 minutos após o término da prova. Essas amostras foram dessoradas e estocadas em "freezer" $\left(-20^{\circ} \mathrm{C}\right)$ até o momento da realização das provas laboratoriais. 
As concentrações séricas de glicose, sódio, potássio, uréia e creatinina foram determinadas nas amostras obtidas de acordo com as seguintes técnicas: glicose: método descrito por HULTMAN (1959), com utilização do reativo Merck $\left(\mathrm{n}^{\circ}\right.$. cat 8313$)$ para glicose; sódio: método de Fotometria de Chama, descrito por KORZUN \& MILLER (1987), em espectrofotômetro de chama da marca Celm, modelo FC-130; potássio: método de Fotometria de Chama, conforme descrito por KORZUN \& MILLER (1987), em espectrofotômetro de chama da marca Celm, modelo FC-130; uréia: técnica da Diacetilmonoxina; creatinina: técnica de LUTSGARTEN \& WENK (1972). Os resultados foram submetidos à análise de variância e o contraste de médias foi analisado através do Teste de Duncan, utilizando-se do programa SAS - Statistical Analysis System (SAS Institute, 1985).

\section{RESULTADOS E DISCUSSÃO}

Os resultados obtidos estão apresentados nas tabelas numeradas de 1 a 5 . A glicose é a principal fonte energética para a contração muscular que ocorre, predominantemente, por via aeróbica, nos animais submetidos a provas longas como o enduro. Apesar de se observar diferença entre os três grupos, em $\mathrm{T}_{1}$, essa diferença não teve significado estatístico. Essas diferenças são, proporcionalmente, mantidas em $\mathrm{T}_{2}$ e $\mathrm{T}_{3}$, o que indica que o consumo de glicose, no decorrer da prova, foi semelhante para os três grupos e que o tempo de reposição das concentrações séricas da glicose não sofre interferência da raça.

Tabela 1 - Avaliação estatística dos contrastes entre médias dos valores de Uréia $(\mathrm{mg} / \mathrm{d} \ell$ ) obtidos nos grupos experimentais formados por equiinos da raça Árabe (Grupo I), da raça Mangalarga (Grupo II) e por animais SRD (Grupo III) participantes da prova de enduro de $30 \mathrm{~km}$, antes, até 30 minutos e até 90 minutos após o final da prova. São Paulo, 1994.

\begin{tabular}{|c|c|c|c|}
\hline Grupos & $\mathrm{T} 1$ & $\mathrm{~T} 2$ & T3 \\
\hline$I$ & $44,18 \mathrm{a}$ & $44,59 \mathrm{a}$ & $45,22 \mathrm{a}$ \\
\hline II & $32,82 \mathrm{c}$ & $40,30 \mathrm{abc}$ & $41,46 \mathrm{ab}$ \\
\hline III & $35,77 \mathrm{bc}$ & $41,51 \mathrm{ab}$ & $44,72 \mathrm{a}$ \\
\hline
\end{tabular}

$\mathrm{T} 1=$ Antes do início da prova.

$\mathrm{T} 2=$ de 20 a 30 minutos após o final da prova.

T3 = de 80 a 90 minutos após o final da prova.

Obs.: Letras diferentes indicam diferença significativa em nível de $\alpha=0,05$.
Tabela 2 - Avaliação estatística dos contrastes entre médias dos valores de Creatinina $(\mathrm{mg} / \mathrm{d} \ell)$ obtidos nos grupos experimentais formados por eqüinos da raça Árabe (Grupo I), da raça Mangalarga (Grupo II) e por animais SRD (Grupo III) participantes da prova de enduro de $30 \mathrm{~km}$, antes, até 30 minutos e até 90 minutos após o final da prova. São Paulo, 1994.

\begin{tabular}{|c|c|c|c|}
\hline Grupos & $\mathrm{T} 1$ & $\mathrm{~T} 2$ & T3 \\
\hline$I$ & $1,953 \mathrm{~b}$ & $2,433 \mathrm{a}$ & $2,480 \mathrm{a}$ \\
\hline II & 1,493 ef & $1,913 \mathrm{bc}$ & $1,673 \mathrm{cde}$ \\
\hline$I I I$ & $1,387 \mathrm{f}$ & $1,793 \mathrm{cbd}$ & $1,647 \mathrm{ed}$ \\
\hline
\end{tabular}

$\mathrm{T} 1=$ Antes do início da prova.

$\mathrm{T} 2$ = de 20 a 30 minutos após o final da prova.

$\mathrm{T} 3$ = de 80 a 90 minutos após o final da prova.

Obs.: Letras diferentes indicam diferença significativa em nível de $\alpha=0,05$.

Com relação às concentrações séricas de glicose em todos os grupos, nos três tempos considerados, observou-se que, em $\mathrm{T}_{2}$, houve redução significativa das médias, quando comparado a $\mathrm{T}_{2}$, e que, em $\mathrm{T}_{3}$, houve aumento da glicemia em relação a $T_{2}$. Esse mesmo tipo de variação foi também relatada por LUCKE \& HALL (1978), SNOW et al. (1982) e OOSTERBAAN et al. (1991), que estudaram equiinos de provas de enduro de 80 , 80 e $100 \mathrm{~km}$ respectivamente, o que, possivelmente, confirma a utilização desse metabólito como fonte energética.

A uréia sérica origina-se da metabolização hepática de compostos nitrogenados e é eliminado do organismo por via renal. $\mathrm{O}$ aumento sérico de uréia não é forte indicativo de lesão renal, porém, como ela tende a seguir passivamente a reabsorção de sódio, que está aumentada quando da redução de fluídos circulantes, pode-se, por meio da dosagem de uréia, avaliar a função renal, visto que os eqüinos submetidos às provas de enduro tendem a apresentar desidratação, além do aumento da gliconeogênese, devido à maior necessidade de energia para manutenção da atividade muscular. No presente trabalho, pôde-se constatar uma diferença das concentrações séricas de uréia para os eqüinos em repouso, decorrente do fator racial, já que os da raça Árabe apresentaram valores de uréia circulante, estatisticamente mais elevados que os Mangalargas e os SRD. Essa diferença, estatisticamente significativa, desapareceu após o exercício, quando os três grupos apresentaram aumento das concentrações sangüíneas de uréia, sendo mais 
Tabela 3 - Avaliação estatística dos contrastes entre médias dos valores de Glicose (mg/d $\ell$ ) obtidos nos grupos experimentais formados por eqüinos da raça Árabe (Grupo I), da raça Mangalarga (Grupo II) e por animais SRD (Grupo III) participantes da prova de enduro de $30 \mathrm{~km}$, antes, até 30 minutos e até 90 minutos após o final da prova. São Paulo, 1994.

\begin{tabular}{|c|c|c|c|}
\hline Tempo & $\mathrm{T} 1$ & $\mathrm{~T} 2$ & $\mathrm{~T} 3$ \\
\hline$I$ & $84,20 \mathrm{ab}$ & $64,80 \mathrm{c}$ & $75,46 \mathrm{bc}$ \\
\hline II & $103,66 \mathrm{a}$ & $76,86 \mathrm{bc}$ & $87,06 \mathrm{ab}$ \\
\hline III & $101,73 \mathrm{a}$ & $74,20 \mathrm{bc}$ & $86,53 \mathrm{ab}$ \\
\hline
\end{tabular}

$\mathrm{T} 1$ = Antes do início da prova.

$\mathrm{T} 2=$ de 20 a 30 minutos após o final da prova.

T3 = de 80 a 90 minutos após o final da prova.

Obs.: Letras diferentes indicam diferença significativa em nível de $\alpha=0,05$.

intenso nos eqüinos da raça Mangalarga e nos SRD, o que determina a diminuição da diferença entre as médias dos três grupos. Não foi encontrado na literatura nenhuma referência à variação dos valores séricos de uréia entre a raça de eqüinos.

ROSE \& HOGDSON (1982) e ROSE $\boldsymbol{e t}$ al. (1983), trabalhando com eqüinos submetidos a treinamento durante algumas semanas (12 e 7 semanas, respectivamente), observaram curvas ascendentes nas concentrações sangüíneas de uréia, não só após o exercício físico, como também durante o repouso. No presente trabalho, acredita-se que variações observadas sejam devido a fatores raciais, pois os eqüinos estudados não foram submetidos a programa de treinamento prévio.

O aumento, estatisticamente significativo da uréia, observado nos animais da raça Mangalarga e nos SRD, durante o enduro e no período de descanso pós-enduro, é semelhante ao descrito por ROSE et al. (1980b) e por SNOW et al. (1982). De acordo com esses autores, essa alteração se deve, principalmente, ao aumento do metabolismo protéico e não à alteração da função renal. $O$ aumento da uréia sérica, não significativo estatisticamente, observado no presente trabalho, nos eqüinos da raça Árabe, também foi descrito por SNOW (1990), nas provas de "três dias". No referido trabalho, SNOW observou que, em uma das provas (Royal Windsor), os eqüinos apresentaram aumento significativo da uréia, enquanto que em outra prova, considerada de maior dificuldade (Brighton), houve aumento, porém, não estatisticamente significativo,que foi atribuido à variabilidade individual dos animais, o que determina melhor adaptação de alguns eqüinos a condições adversas.
A creatinina sérica, a exemplo do que ocorre com a uréia, sofre influências de condições pré-renais, como intensa atividade ou alteração muscular e, também, devido à hipovolemia que leva à diminuição da filtração glomerular. No presente trabalho, observou-se, para eqüinos em repouso, diferença estatisticamente significativa entre os três grupos considerados, sendo que nos eqüinos da raça Árabe o valor médio de creatinina sérica foi maior do que o da raça Mangalarga e nos SRD. Não foi encontrado na literatura compilada informações quanto à diferença de níveis séricos de creatinina, determinada por fator racial, porém, constata-se que, no presente estudo, esse foi o fator determinante dessa diferença.

Nos três grupos, evidenciou-se aumento da creatinina, de intensidade semelhante em $\mathrm{T}_{2}$, e diminuição dos mesmos em $\mathrm{T}_{3}$, ou seja, após sessenta minutos de descanso. Resultados semelhantes foram obtidos por ROSE $\boldsymbol{e t}$ al. (1982), SNOW et al. (1983) e ROSE et al. (1980a), tanto em provas de enduro como nas chamadas provas de três dias. Nas provas de enduro, ROSE \& HODGSON (1982) também observaram aumento da ordem de $30 \%$ nas concentrações de creatinina circulante, com discreta redução, após trinta minutos de repouso, e retorno às concentrações normais após dezoito horas de descanso. ROSE \& HODGSON (1982), que trabalharam com eqüinos mestiços da raça Árabe, em programa de treinamento para enduro, durante doze semanas, observaram grande variação da creatinina sérica nos eqüinos em repouso, porém foi semelhante ao obtido no presente trabalho, para o grupo de eqüinos SRD, ou seja, abaixo dos valores apresentados para os da raça Árabe.

Tabela 4 - Avaliação estatística dos contrastes entre médias dos valores Sódio $(\mathrm{mEq} / \ell)$ obtidos nos grupos experimentais formados por eqüinos da raça Árabe (Grupo I), da raça Mangalarga (Grupo II) e por animais SRD (Grupo III) participantes da prova de enduro de $30 \mathrm{~km}$, antes, até 30 minutos e até 90 minutos após o final da prova. São Paulo, 1994.

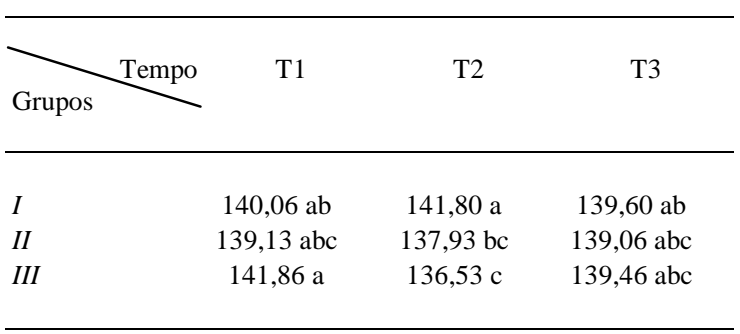

$\mathrm{T} 1=$ Antes do início da prova.

$\mathrm{T} 2$ = de 20 a 30 minutos após o final da prova.

T3 = de 80 a 90 minutos após o final da prova.

Obs.: Letras diferentes indicam diferença significativa em nível de $\alpha=0,05$.

Ciência Rural, v. 30, n. 3, 2000. 
Tabela 5 - Avaliação estatística dos contrastes entre médias dos valores de Potássio $(\mathrm{mEq} / \ell)$ obtidos nos grupos experimentais formados por eqüinos da raça Árabe (Grupo I), da raça Mangalarga (Grupo II) e por animais SRD (Grupo III) participantes da prova de enduro de $30 \mathrm{~km}$, antes, até 30 minutos e até 90 minutos após o final da prova. São Paulo, 1994.

\begin{tabular}{|c|c|c|c|}
\hline Grupos & $\mathrm{T} 1$ & $\mathrm{~T} 2$ & T3 \\
\hline$I$ & $3,680 \mathrm{ab}$ & $2,613 \mathrm{~cd}$ & $2,993 \mathrm{c}$ \\
\hline II & $3,533 \mathrm{~b}$ & $2,673 \mathrm{~cd}$ & $2,480 \mathrm{~d}$ \\
\hline III & $3,907 \mathrm{a}$ & $3,067 \mathrm{c}$ & $2,827 \mathrm{~cd}$ \\
\hline
\end{tabular}

$\mathrm{T} 1=$ Antes do início da prova.

$\mathrm{T} 2$ = de 20 a 30 minutos após o final da prova.

T3 = de 80 a 90 minutos após o final da prova.

Obs.: Letras diferentes indicam diferença em nível de $\alpha=0,05$.

Notou-se diferença de comportamento dos valores médios de sódio sérico dos eqüinos da raça Árabe, em relação aos demais eqüinos, pois, nos Árabes, a média obtida em $\mathrm{T}_{2}$ foi superior à de $\mathrm{T}_{1} \mathrm{e}$ $T_{3}$, enquanto nos outros grupos, a média de $T_{2}$ foi inferior às médias de $\mathrm{T}_{1}$ e $\mathrm{T}_{3}$; essas variações não foram estatisticamente significativas, mas mostram a tendência do comportamento do sódio. Variação semelhante, para os eqüinos da raça Árabe, foram descritas também por ROSE et al. (1980b), SNOW et al. (1982), ROSE et al. (1983), SNOW (1990) e OOSTERBAAN et al (1991).

LEAF (1962) afirmou que a elevação dos níveis de sódio sérico é indicativo da diminuição de fluido extracelular, portanto, o aumento da concentração sérica em $\mathrm{T}_{2}$, nos eqüinos da raça Árabe, pode ser reflexo de maior perda de fluidos circulantes, quando comparados aos dos outros dois grupos raciais, o que acarretaria mobilização mais rápida de sódio, na tentativa de manutenção do equilíbrio osmótico, ainda durante o exercício, como consta no trabalho de OOSTERBAAN et al. (1991).

A diminuição da concentração sangüínea de sódio, observada nos eqüinos da raça Mangalarga e nos SRD, após o exercício, também foi relatada por LUCKE \& HALL (1978) e por ROSE $\boldsymbol{e} \boldsymbol{t} \boldsymbol{a l}$. (1980b), em eqüinos de enduro, atribuindo essa diminuição do sódio à perda desse elemento pelo suor. A concentração sérica de sódio pode sofrer influência do manejo e do treinamento, conforme demonstraram MULLEN et al. (1979) e ROSE \& HODGSON (1982), porém esses fatores não devem ter influenciado os resultados do presente trabalho, visto que eram eqüinos submetidos a manejo semelhante e que não faziam programa de treinamento prévio.

A alteração da concentração sérica de potássio foi semelhante nos três grupos estudados, ou seja, houve redução significativa em $T_{2}$ e $T_{1}$. Porém, ao comparar as concentrações sangüíneas médias de $\mathrm{T}_{3}$ às de $\mathrm{T}_{2}$, observou-se que a resposta é diferente nos eqüinos da raça Árabe, que apresentam início da reposição do potássio circulante, enquanto nos outros grupos raciais ainda ocorre diminuição do potássio sérico. Resultados semelhantes aos obtidos no presente trabalho foram descritos por WILLIANSON (1974), LUCKE \& HALL (1978), ROSE et al. (1980b), SNOW et al. (1982) e ROSE et al. (1983). Esses autores atribuem a diminuição do potássio, principalmente, à perda deste elemento pelo suor. ROSE et al. (1980b) OOSTERBAAN $\boldsymbol{e} t$ al (1991) observaram aumento de potássio sérico, enquanto SNOW (1990) obteve valores médios de potássio semelhantes nas amostras colhidas antes e após o exercício. Esses resultados, aparentemente conflitantes com relação ao potássio sérico, provavelmente ocorreram devido às diferenças climáticas e dos próprios percursos em que foram realizados as diferentes provas.

Outro fator a ser considerado é que as maiores reservas de potássio no organismo são intracelulares, o que acarreta um maior tempo para mobilização desse elemento para a corrente circulatória, a fim de que sejam restabelecidas as concentrações iniciais. ROSE \& HODGSON (1982) afirmaram que as concentrações séricas de potássio tendem a declinar, quando os animais são submetidos a treinamento constante. Acredita-se que o comportamento diferente observado entre os equiinos da raça Mangalarga e os SRD não se deva a esse fator e nem à sazonalidade, conforme descreveram MULLEN et al. (1979), visto que as amostras utilizadas neste trabalho foram colhidas de forma homogênea, em relação ao tempo, e de animais que não estavam sendo submetidos a treinamento específico.

\section{CONCLUSÕES}

A variação das concentrações séricas de glicose, potássio e creatinina ocorre de forma semelhante nos três grupos de eqüinos quando se considera o tempo de colheita. A variação das concentrações séricas de uréia só é significante nos grupos compostos por eqüinos Mangalarga e nos $\mathrm{SRD}$, e a variação das concentrações séricas de sódio nos eqüinos da raça Árabe não apresenta o mesmo padrão observado nos outros grupos. 


\section{REFERÊNCIAS BIBLIOGRÁFICAS}

HULTMAN, E. Rapid specific method for determination of aldosaccharides in the body fluids. Nature, v.183, p.108, 1959.

KORZUN, W.J., MILLER, W.G. Sodium and potassium. In: PESCE, A.J., KAPLAN, L.A.. Methods in clinical chemistry. Saint Louis : Mosby, 1987. p.86-91.

LEAF, A. The clinical and physiologic significance of the serum sodium concentration. New Eng J Med, v.267, p.24-30, 1962.

LUCKE, J.N., HALL, G.M. Biochemical changes in horses during a 50-mile endurance ride. Vet Rec, v.102, n.16, p.356358, 1978.

LUTSGARTEN, J.A., WENK, R.E. Simple, rapid, kinetic method for serum creatinin measurement. Clinical Chemistry, v.18, n.11, p.1419-22, 1972.

MULLEN, P.A., HOPES, R., SEWELL, J. The biochemistry, haematology, nutrition and racing performance of two-yearold thoroughbreds throughout their training and racing season. Vet Rec, v.104,n.5, p.90-95, 1979.

OOSTERBAAN, M.M.S.O., WENSING, T., BARNEVELD, A., et al. Heart rate, blood biochemistry and performance of horses competing in a $100 \mathrm{~km}$ endurance ride. Vet Rec, v.128, n.8, p.175-179, 1991.

ROSE, R.J., BACKHOUSE, J.W., ILKIW, J.E. Electrocardiography and haematology of horses competing in a three-day event. Aust Vet J, v.56, n.7, p.318-320, 1980a.

ROSE, J.R., ARNOLD, K.S., CHURCH, S., et al. Plasma and sweat electrolyte concentrations in the horse during long distance exercise. Equine Vet J, v.12, n.1, p.19-22, 1980 b.

ROSE, R.J., HODGSON, D.R. Haematological and plasma biochemical parameters in endurance horses during training. Equine Vet J, v.14, n.2, p.144-148, 1982.

ROSE, R.J., ALLEN, J.R., HODGSON, D.R., et al.. Responses to submaximal treadmill exercise and training in the horse: changes in haematology, arterial blood gas and acid base measurements, plasma biochimical values and heart rate. Vet Rec, v.113, n 26-27, p.612-618, 1983.

SAS Institute. SAS user's guide: statistics. 1985 ed. Cary : SAS Institute, 1985. 956p.

SNOW, D.H. Haematological, biochemical and physiological changes in horses and ponies during the cross country stage of driving trial competitions. Vet Rec, v.126, n.10, p.233-239, 1990 .

SNOW, D.H., KERR, M.G., NIMMO, M.A., et al. Alterations in blood, sweat, urine and muscle composition during prolonged exercise in the horse. Vet Rec, v.110, n.16, p.377-384, 1982.

SNOW, D.H., RICKETTS, S.W., MASON, D.K. Haematological response toracing and training exercise in thoroughbred horses, with particular reference to the leucocyte response. Equine Vet J, v.15, n.2, p.149-54, 1983.

WILLIAMSON, H.M. Normal and abnormal electrolyte levels in the racing horse and their effect on performance. J South African Vet Assoc, v.45, n.4, p.335-340, 1974.

Ciência Rural, v. 30, n. 3, 2000. 
Ciência Rural, v. 30, n. 3, 2000. 